\title{
Physical Activity and Quality of Life Improvements of Patients With Idiopathic Pulmonary Fibrosis Completing a Pulmonary Rehabilitation Program
}

\author{
Ignacio A Gaunaurd PT PhD, Orlando W Gómez-Marín MSc PhD, Carol F Ramos, \\ Constanza M Sol MSc, Meryl I Cohen PT DPT, Lawrence P Cahalin PT PhD, \\ Diana D Cardenas MD, and Robert M Jackson MD
}

\begin{abstract}
INTRODUCTION: Pulmonary rehabilitation is effective for patients with COPD, but its benefit is less clearly established in idiopathic pulmonary fibrosis (IPF), especially in regard to levels of physical activity and health-related quality of life. The objectives were to determine whether pulmonary rehabilitation increased physical activity as assessed by the International Physical Activity Questionnaire (IPAQ), and improved quality of life and symptoms as assessed by the St George respiratory questionnaire for IPF (SGRQ-I) and the Borg dyspnea index (BDI). METHODS: Subjects who met current criteria for IPF were randomized to a 3-month pulmonary rehabilitation program $(n=11)$ or to a control group $(n=10)$. The rehabilitation group participated in twiceweekly, 90-min exercise sessions ( 24 total sessions). The control group maintained its preceding, normal physical activity. All subjects underwent 6-min walk tests to assess the postexertion BDI. The SGRQ-I and a 5-point self-assessment of health were completed at baseline, after 3 months of intervention or observation, and after 3-month follow-up. All subjects completed the IPAQ weekly. RESULTS: Subjects in the rehabilitation group maintained significantly higher levels of physical activity throughout the 3 -month rehabilitation program (rehabilitation: 51,364 $\pm 57,713$ [mean \pm SD] metabolic equivalent of task-minutes; control: $20,832 \pm 37,155, P=.027$ by 2 -tailed Mann-Whitney test). SGRQ-I symptom domain scores improved considerably by $-9 \pm 22$ in the rehabilitation group, whereas in the control group they worsened (16 \pm 12 rehabilitation compared with control, $P=.013$ by 2-tailed Mann-Whitney test). During the 3-month follow-up, self-reported physical activity levels in the rehabilitation group were 14,428 $\pm 8,884$ metabolic equivalent of task-minutes and in the control group 16,923 $\pm 32,620(P=.17$ by 2-tailed Mann-Whitney test), demonstrating substantial reversal of activity in the rehabilitation group. BDI scores after 6-min walk tests did not change significantly. CONCLUSIONS: A 3-month rehabilitation program significantly improved symptoms (SGRQ-I) and physical activity levels (IPAQ) in subjects with IPF while they participated actively in the program. (ClinicalTrials.gov registration NCT01118221.) Key words: idiopathic pulmonary fibrosis; pulmonary rehabilitation; exercise; quality of life. [Respir Care 2014;59(12):1872-1879. ( 2014 Daedalus Enterprises]
\end{abstract}

\section{Introduction}

Physical activity and quality of life improvements in patients with COPD who have participated in pulmonary rehabilitation are well documented. ${ }^{1}$ Such clear benefits

Drs Gaunaurd and Gómez-Marín, Ms Ramos, Ms Sol, and Drs Cardenas and Jackson are affiliated with the Research Service, Miami Veterans have not been reported in patients with interstitial lung diseases (ILD) and, in particular, not in patients with id-

\footnotetext{
Affairs Healthcare System; Dr Gómez-Marín is also affiliated with the Departments of Public Health Sciences, Pediatrics, and Medicine, University of Miami; Ms Cohen and Dr Cahalin are affiliated with the Department of Physical Therapy, University of Miami; and Dr Cardenas is also affiliated with the Department of Physical Medicine and Rehabilitation, University of Miami, Miami, Florida.
} 
iopathic pulmonary fibrosis (IPF), the most common and severe fibrotic ILD. ${ }^{2}$ IPF is usually progressive and poorly amenable to therapy. ${ }^{3}$ In a limited number of studies, physical training in the form of pulmonary rehabilitation appeared not to improve dyspnea in patients with various ILD, and quality of life improved only marginally after training. ${ }^{4}$ Additional investigation is needed to determine benefits of pulmonary rehabilitation, if any, for patients with ILD, especially for those with IPF in regard to its effects on physical activity and quality of life. ${ }^{5}$ No previous studies have examined effects of pulmonary rehabilitation on the level of physical activity in carefully selected subjects with IPF.

We designed a pulmonary rehabilitation program for patients with IPF. We then investigated both mechanisms of exercise limitation and possible beneficial effects of physical training on sustained and peak exercise. The goals of this pilot study were to determine how a 3-month, twice weekly pulmonary rehabilitation program influenced physical activity and quality of life of patients with IPF, and whether these effects persisted during a subsequent 3-month observation period. We used the International Physical Activity Questionnaire (IPAQ) to estimate physical activity levels and the St George Respiratory Questionnaire for IPF (SGRQ-I) at baseline, after the 3-month intervention (rehabilitation) or observation period (control), and 3 subsequent months following the intervention period. ${ }^{6,7} \mathrm{We}$ hypothesized that differences in physical activity, quality of life, and postexertional dyspnea would be found between the rehabilitation and control groups.

\section{Methods}

\section{Study Participants}

The Miami Veterans Affairs Health System Institutional Review Board approved this study. IPF cases were defined according to American Thoracic Society-European Respiratory Society clinical diagnostic criteria. ${ }^{8}$ Inclusion and exclusion criteria were identical to those described else-

This research was supported by a Merit Review Award from the Department of Veterans Affairs Research Service.

Drs Gaunaurd and Gómez-Marín contributed equally to this work.

Dr Gaunaurd presented preliminary findings at the 2013 American Thoracic Society International Conference in Philadelphia, PA.

The authors have disclosed no conflicts of interest.

Correspondence: Robert M Jackson MD, Research Service (151), 1201 NW 16th Street, Miami, FL 33125. E-mail: rjackson2@med.miami.edu.

DOI: $10.4187 /$ respcare 03180

\section{QUICK LOOK}

\section{Current knowledge}

Pulmonary rehabilitation is a comprehensive intervention including education, training, and behavior modification aimed at improving quality of life in patients with chronic respiratory disease. Pulmonary rehabilitation has been effective in improving quality of life in COPD, but has not been widely studied in IPF.

\section{What this paper contributes to our knowledge}

A pulmonary rehabilitation program benefits patients with IPF by enhancing their level of physical activity while in the program and by significantly reducing disease burden. The long-term impact was not evaluated.

where. ${ }^{9,10}$ To screen and define cases, we consistently used the following diagnostic scheme. Three major criteria were required, including exclusion of other known causes of ILD, abnormal pulmonary function tests, and bibasilar, reticular abnormalities with minimal ground glass opacities on high-resolution computed tomography. We also required 3 of the 4 following minor criteria: age over $50 \mathrm{y}$, insidious onset of dyspnea, duration of symptoms over 3 months, and bibasilar, inspiratory Velcro-like crackles. ${ }^{8}$ All enrolled subjects were ambulatory out-patients.

Patients were screened by echocardiography at rest to estimate right ventricular systolic pressure. ${ }^{11}$ High-resolution computed tomographic scans of the chest were required to show a usual interstitial pneumonia pattern. ${ }^{12}$ Eligible subjects were assigned by block randomization to either the rehabilitation or control group according to a random number program. The distribution of subjects screened and randomized is summarized in Figure 1.

\section{Experimental Design}

Twenty-one subjects were randomly assigned to undergo either a 3-month pulmonary rehabilitation program (rehabilitation group $=11$ ) or no organized exercise (control group $=10$ ), after which both groups were followed for an additional 3 months. At each time period's end, a 6-min walk test (6MWT), cycle ergometry, SGRQ-I with health self-assessment, and IPAQ were done. All subjects completed the IPAQ weekly. Control subjects $(n=10)$ were evaluated at the same time points, but they did not participate in organized exercise.

\section{6-min Walk Test Protocol}

The 6MWT was done according to American Thoracic Society guidelines. ${ }^{13}$ If subjects used supplemental oxygen 


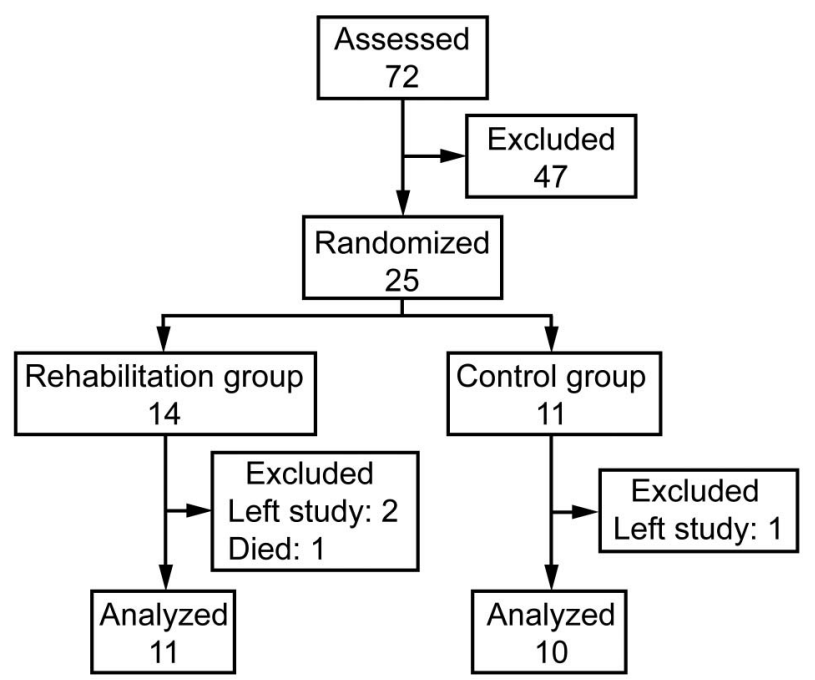

Fig. 1. Selection and disposition of subjects. We screened 72 IPF patients for eligibility and enrolled 25 . Fourteen were assigned to rehabilitation and 11 to the control (observation only) group. Two rehabilitation subjects and 1 control subject chose to leave the trial. One rehabilitation subject died of respiratory failure during the trial. Twenty-one subjects completed the trial, and their data are presented here.

at the initial evaluation, they used the same level of supplemental oxygen during each subsequent test. Heart rate, blood pressure, $\mathrm{S}_{\mathrm{pO}_{2}}$, and Borg dyspnea index (BDI) were recorded. ${ }^{14}$

\section{Pulmonary Function Tests}

Pulmonary function tests were done in the Pulmonary Function Laboratory of the Miami Veterans Affairs Healthcare System using clinically certified, computerized spirometry and plethysmography equipment.

\section{Rehabilitation Program}

The rehabilitation group participated in a rigorously structured, 12-week pulmonary rehabilitation program that included educational lectures and supervised aerobic and strengthening exercises. Subjects participated in 2 90-min sessions per week and were required to complete all 24 treatment sessions. Ten educational lectures were provided throughout the 12 -week program, including medication use, breathing techniques, exercise training, nutrition, pulmonary physiology, and psychological coping mechanisms among others. Handouts were provided to the control subjects so they could read the lecture content.

Supervised exercise included $30 \mathrm{~min}$ of cardiopulmonary endurance training, $20 \mathrm{~min}$ of flexibility exercises, and 25 min of strength training. Cardiopulmonary endurance training reached targeted goals of $20 \mathrm{~min}$ of treadmill walking (Life Fitness Club Series treadmill) and 10 min of recumbent cycling (Lifecycle 9500 exercise bike). The intensity of the endurance training was set at $70-80 \%$ of the maximum predicted heart rate for each subject. The total metabolic equivalent of task minutes (MET-min) for treadmill and cycle exercise were calculated at each session. ${ }^{15}$ Participants progressed as tolerated from interval conditioning to walking or cycling continuously for the targeted time. $\mathrm{S}_{\mathrm{pO}_{2}}$, BDI, and vital signs, including heart rate and blood pressure, were monitored and recorded during aerobic conditioning.

Participants receiving supplemental oxygen therapy before study enrollment used oxygen during the rehabilitation sessions. Supplemental oxygen was provided from a compressed source as needed during exercise sessions to maintain $\mathrm{S}_{\mathrm{pO}_{2}}$ above $88 \%$. Short rest intervals were taken when $\mathrm{S}_{\mathrm{pO}_{2}}$ level fell below $88 \%$.

Stretches were designed to target musculoskeletal restrictions typical of a chronically ill population. ${ }^{16}$ These consisted of corner stretches for the pectoral muscles, lower cervical and upper thoracic stretches for cervical spine and upper back muscles, thoracolumbar side bends for lateral trunk flexors and intercostal muscles, knee extension stretches for hamstring muscles, standing knee flexion for quadriceps muscles, and standing forward lunges for gastrocnemius muscles. Stretches were held for at least $30 \mathrm{~s}$ 3 times at each session.

Strength training targeted major muscle groups. Upper body exercises were done during first and second treatment sessions and lower body exercises during the third and fourth sessions. The first session included sitting or standing biceps curls, mid-back rowing, and shoulder flexion. Sitting or standing chest presses and triceps extensions were done during the second session. Lower body exercises during the third included standing hip abduction and extension. The fourth included seated knee flexion and extension. The 4 sessions were then repeated. Subjects began strength training using a yellow therapeutic band while doing 2 sets of 10 repetitions for each exercise and progressing to 3 sets of 15 . Strength training was advanced to 3 sets of 15 repetitions from a red therapeutic band and to a green band.

A home exercise program and therapeutic bands were provided to participants in the rehabilitation group. The physical therapist instructed them to exercise at home twice a week on days they did not do pulmonary rehabilitation.

\section{Data Collection and Outcome Measures}

The IPAQ short form version was completed weekly by each subject throughout the 6-month period to record physical activity over the prior $7 \mathrm{~d}$. The IPAQ records the number of days per week of vigorous or moderate activity, walking and sitting, and the time of each per day. ${ }^{6}$ IPAQ 
results were compiled at baseline, following the 3-month rehabilitation or observation (controls) and the subsequent 3-month follow-up period.

A 5-point scale for self-assessment of health and the SGRQ-I were completed at baseline, after pulmonary rehabilitation or observation (controls), and again after the subsequent 3-month follow-up, to assess quality of life and effects of rehabilitation on symptoms, activity, and impact on health. ${ }^{7}$ The self-assessment of health consisted of a scale with responses coded from 1 to 5 corresponding to very poor, poor, fair, good, or very good.

We recorded the BDI before and after the 6MWT at 0 , 3 , and 6 months to assess the effects of physical training on breathlessness and whether effects persisted during a subsequent 3-month observation period after the rehabilitation program. ${ }^{14}$

\section{Statistical Analyses}

Data are presented as arithmetic means \pm SD unless noted. Paired $t$ tests or Wilcoxon signed rank tests were used for within group comparisons. Independent sample $t$ tests or Mann-Whitney tests were used for between group comparisons when examining 6MWT, SGRQ-I, IPAQ, and postexertion BDI scores at baseline, after 3 months of rehabilitation or observation periods, and after subsequent 3-month follow-up. IPAQ MET-min were calculated using the IPAQ short form scoring guidelines. ${ }^{6}$ A generalized linear model was used to analyze trends in IPAQ area under the curve MET-min. ${ }^{17}$ Statistical significance was defined as $P<.05$. SAS 9.3 (SAS Institute, Cary, North Carolina) and SigmaPlot 12 (Systat Software, San Jose, California) were used for data analysis. All statistical tests were 2-sided and conducted at a nominal 5\% level of significance.

\section{Results}

\section{Characteristics and Disposition of the Subjects}

All subjects at enrollment met American Thoracic Society-European Respiratory Society criteria for IPF. ${ }^{8}$ Comorbidities were thus limited to common, non-life-threatening conditions that occur in this age group. Eleven subjects in the rehabilitation group and 10 in the control group completed the entire 6-month study and were analyzed and reported here. The participants' baseline characteristics are presented in Table 1.

At enrollment, subjects had mild to moderate restriction of lung volumes (mean FVC $61 \pm 12 \%$ of predicted) and moderate to severe diffusion impairment (mean diffusing capacity for carbon monoxide $\left[\mathrm{D}_{\mathrm{LCO}}\right] 44 \pm 10 \%$ of predicted).
Table 1. Baseline Characteristics of Study Participants by Group

\begin{tabular}{|c|c|c|c|}
\hline Variable & $\begin{array}{l}\text { Rehabilitation } \\
\text { Group* } \\
(n=11)\end{array}$ & $\begin{array}{l}\text { Control } \\
\text { Group** } \\
(n=10)\end{array}$ & $P$ \\
\hline Age (y) & $71 \pm 6$ & $66 \pm 7$ & .09 \\
\hline Weight (kg) & $97 \pm 20$ & $92 \pm 21$ & .58 \\
\hline Height (cm) & $175 \pm 10$ & $166 \pm 12$ & .08 \\
\hline FVC (\% predicted) & $60 \pm 11$ & $61 \pm 14$ & .86 \\
\hline $\mathrm{FEV}_{1} / \mathrm{FVC}$ (ratio) & $0.84 \pm 0.04$ & $0.86 \pm 0.05$ & .69 \\
\hline TLC (\% predicted) & $58 \pm 8$ & $60 \pm 12$ & .66 \\
\hline $\mathrm{D}_{\text {LCO }}(\%$ predicted $)$ & $44 \pm 11$ & $43 \pm 11$ & .84 \\
\hline MVV (L/min) & $69 \pm 16$ & $75 \pm 26$ & .53 \\
\hline $\mathrm{S}_{\mathrm{pO}_{2}}(\%)$ & $95 \pm 3$ & $96 \pm 2$ & .39 \\
\hline 6MWT distance $(\mathrm{m})$ & $361 \pm 55$ & $339 \pm 109$ & .58 \\
\hline RVSP (mm Hg) & $33 \pm 17$ & $37 \pm 18$ & .61 \\
\hline $\begin{array}{l}\text { Data are means } \pm \text { SD. } \\
* \text { The control group did nor } \\
\text { ** The rehabilitation group } \\
\text { program. } \\
\text { TLC = total lung capacity } \\
\text { MVV = maximum voluntar } \\
6 \mathrm{MWT}=6 \text {-min walk test } \\
\text { RVSP = right ventricular sy }\end{array}$ & $\begin{array}{l}\text { vities for } 3 \text { mo. } \\
\text { ed a } 12-w k \text {, twice wh } \\
\text { ation } \\
\text { ressure }\end{array}$ & ulmonary rehabilit & \\
\hline
\end{tabular}

We carefully excluded patients with IPF who had decompensated heart failure, severe pulmonary hypertension, and diseases expected to shorten their predicted survival, such as lung cancer. A number of common comorbidities occurred in the study population including (in decreasing frequency) hypertension (12 of 21), gastroesophageal reflux disease (9), hyperlipidemia (9), diabetes mellitus (5), depression (2), and history of heart disease (1). The comorbidities were relatively evenly distributed between the control and rehabilitation groups.

\section{SGRQ-I}

Subjects completed the SGRQ-I initially, after 3-month rehabilitation or control group participation, and again after a subsequent 3-month observation. Those who completed the 12-week rehabilitation program experienced a clinically important improvement in the symptoms domain score compared with the controls, who experienced significant worsening $(P=.01)$. These data are summarized and presented in Table 2. Otherwise, activity, impact, and total scores did not change significantly after the 3-month rehabilitation program.

As shown in Table 3, at baseline, 3-month, and 6-month visits significant negative Spearman rank correlations ranging from -0.26 to -0.53 were observed between self-reported heath scores, SGRQ-I domain scores and the total scores, when all enrolled subjects were considered together. 
Table 2. Effects of Pulmonary Rehabilitation on Changes in SGRQI Symptom Scores

\begin{tabular}{lccc}
\hline \hline & $\begin{array}{c}\text { Rehabilitation } \\
(n=11)\end{array}$ & $\begin{array}{c}\text { Control } \\
(n=10)\end{array}$ & $P^{*}$ \\
\hline 3 mo compared to 0 mo & & & \\
Mean \pm SD & $-9.1 \pm 22.2$ & $15.5 \pm 12.3$ & .01 \\
$95 \%$ CI & -24.0 to 5.9 & 6.6 to 24.3 & \\
$P^{* *}$ & .262 & .014 & \\
6 mo compared to 3 mo & & & \\
Mean \pm SD & $7.2 \pm 16.3$ & $-13.4 \pm 28.0$ & .06 \\
$95 \%$ CI & -3.8 to 18.1 & -33.5 to 6.5 & \\
$P^{* *}$ & .205 & .036 \\
& & \\
* Mann-Whitney test 2-tailed $P$ value: rehabilitation compared to control. \\
** Normal approximation to Wilcoxon signed rank test 2-tailed $P$ value for within group \\
changes. \\
SGRQ-I St George respiratory questionnaire for idiopathic pulmonary fibrosis \\
\end{tabular}

Table 3. Self-Assessment of Health (0-5) Correlates With SGRQ-I Scores

\begin{tabular}{lccc}
\hline \hline \multirow{3}{*}{ SGRQ-I Module } & \multicolumn{3}{c}{$\mathrm{R}^{*}(P)^{* * *}$} \\
\cline { 2 - 4 } & Baseline & $3 \mathrm{mo}$ & 6 mo \\
\hline Activity & $-0.40(.077)$ & $-0.62(.003)$ & $-0.37(.126)$ \\
Impact & $-0.69(.001)$ & $-0.66(.002)$ & $-0.40(.098)$ \\
Symptoms & $-0.43(.050)$ & $-0.58(.007)$ & $-0.46(.053)$ \\
Total & $-0.59(.006)$ & $-0.67(.001)$ & $-0.52(.027)$ \\
& & \\
Data include all enrolled subjects $(N=21)$. \\
* Spearman rank correlation coefficients for health self-assessment score and SGRQ-I scores. \\
** Corresponding $P$ values are shown in parentheses. \\
SGRQ-I = St George respiratory questionnaire for idiopathic pulmonary fibrosis \\
\hline
\end{tabular}

\section{IPAQ}

We used self-reported physical activity during each week of the study to calculate cumulative weekly MET-min exerted by each participant. ${ }^{16}$ We calculated areas under the curves from 0 to 3 months and from 3 to 6 months to compare effects of the rehabilitation program on activity and determine whether it persisted after rehabilitation.

During the first 3-month period, subjects participating in the rehabilitation program had significantly greater selfreported physical activity $(51,364 \pm 57,713$ MET-min $)$ than did controls $(20,892 \pm 37,155, P=.03$ by 2 -tailed Mann-Whitney test). During the subsequent 3-month observation period (ie, after completion of rehabilitation), subjects in both groups reverted to similar physical activity levels (rehabilitation $14,428 \pm 8,884$; controls $16,793 \pm 32,620, P=.17$ by 2 -tailed Mann-Whitney test). These data are shown in Figure 2. Similar changes were found when moderate physical activity was considered alone. No significant differences were found when walking or vigorous physical activities were compared separately.

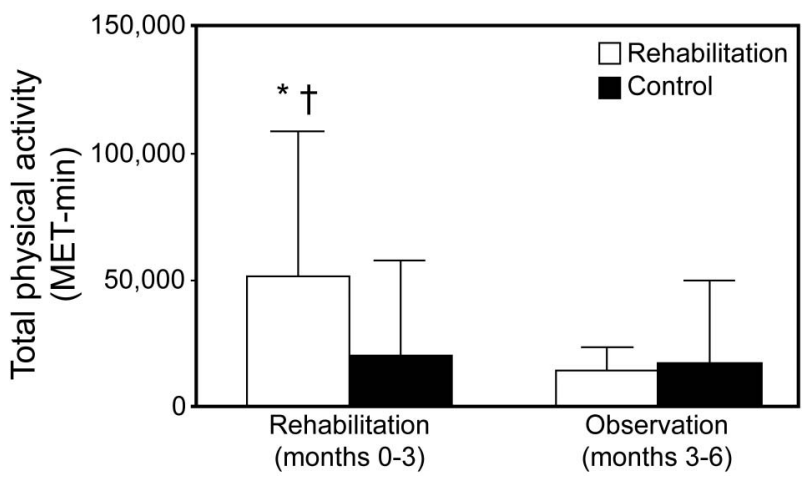

Fig. 2. Self-reported total physical activity during pulmonary rehabilitation and subsequent 3-month observation period. We assessed levels of total physical activity subjectively by having subjects complete the IPAQ short form weekly during the initial 3-month pulmonary rehabilitation period, and subsequently during a 3-month observation period. Both control and rehabilitation subjects completed the questionnaire by telephone or during rehabilitation sessions, respectively. Physical activity levels were quantified and expressed as areas under the curve generated by plotting the total metabolic equivalent of task-minutes (MET-min) reported and then integrating with respect to time over each 3-month epoch. Subjects participating in pulmonary rehabilitation had significantly higher self-reported levels of physical activity compared with controls (2.5-fold) during the initial 3 months. However, this increase was not sustained during the subsequent 3-month observation period, when both groups reported nearly identical levels of activity. ${ }^{*} P=.02$ compared with controls during months $0-3 .{ }^{\star}{ }^{\star} P=.01$ compared with the 3-6-month rehabilitation level.

\section{Post-Rehabilitation Physiology}

Total lung capacity, FVC, and $\mathrm{D}_{\mathrm{LCO}}$ did not change significantly in either group over the 6-month study period. However, the $\mathrm{D}_{\mathrm{LCO}}$ tended to decrease over that period only in the control group (exploratory analysis by one-tailed, paired $t$ test showed that $P=.04$ for the baseline $\mathrm{D}_{\mathrm{LCO}}$ compared with the 6-month $\mathrm{D}_{\mathrm{LCO}}$ ).

\section{Discussion}

Symptoms limit activity and impair the quality of life of patients with IPF. No effective pharmacotherapy for IPF exists, so improvement in quality of life is needed and avidly sought by patients. Dyspnea is the cardinal symptom of IPF, and it may be disabling at low activity levels. Weakness, presumably due to skeletal muscle dysfunction, might also limit the ability of IPF patients to do physical tasks. ${ }^{18}$ Relief of such symptoms would improve quality of life and enhance physical activity.

We specifically tested whether a 3-month pulmonary rehabilitation program increased physical activity levels, as shown by the IPAQ short form, and improved IPF patients' quality of life, as measured by the SGRQ-I. We studied 21 well-characterized subjects, all of whom met 
contemporary American Thoracic Society-European Respiratory Society criteria for IPF. The study population is not directly comparable to other studies, which often include subjects with other interstitial lung diseases. ${ }^{4}$

IPF presents a unique therapeutic challenge in that it is usually progressive and occurs commonly in older individuals. Physical training of patients with IPF will likely require specifically targeted approaches that take into account the rapid development of hypoxemia during exercise, limiting dyspnea, and skeletal muscle dysfunction that result from tissue hypoxia and systemic oxidant stress. ${ }^{10,16}$

Improvement of quality of life is clearly important for patients with IPF, since both physical health and level of independence are negatively impacted by the disease. These might be worsened by the presence of depression, ${ }^{19}$ as dyspnea appears related both to the quality of life and degree of depression. Because we did not use a specific measure of depression, we do not comment on the effect, if any, of depression on our results.

When assessed by the Short Form-36 (SF-36) for measuring health-related quality of life, worsening correlates with lower vital capacity and 6-min walk distance. ${ }^{20}$ Males tend to be more severely impacted by dyspnea than females in regard to quality of life. ${ }^{21}$ Although pulmonary function and oxygenation were each correlated with the SGRQ score in 41 patients with IPF, dyspnea was the most prominent variable (based on regression analysis) contributing to impaired quality of life. ${ }^{5}$

We used a version of the SGRQ specific for IPF (SGRQ-I) to assess subjects' subjective wellbeing and response to exercise training. This version eliminates a number of items not relevant to IPF, and it encompasses a new scoring scheme. It correlates well with the original SGRQ, while focusing on items more relevant to IPF. ${ }^{7}$ Over the 3 -month study period, symptoms decreased in the rehabilitation subjects, whereas they increased in the control subjects. These changes were both clinically important and statistically significant based on the accepted minimum important difference for SGRQ scores. We found that a simplified 5-point scale provided similar information regarding perceived health. Participation in the rehabilitation program had a beneficial effect in regard to decreasing symptoms.

Pulmonary rehabilitation of patients with IPF minimizes specific IPF-related symptoms, but it does not produce a global improvement in health related quality of life. ${ }^{22,23,24}$ Meta-analysis revealed a small beneficial effect of pulmonary rehabilitation on quality of life immediately after training; it appeared to not persist over the long term, making our results consistent with the literature. ${ }^{4}$ After 6 months, no differences in symptom scores were present.

Next, we assessed whether pulmonary rehabilitation increased physical activity levels of subjects. Rehabilitation subjects clearly had significantly increased physical activ- ity levels during the initial 3-month period (see Fig. 2). Self-reported activity during the time included activities at home and during rehabilitation sessions. An increase in physical activity occurred despite no measurable change in dyspnea. Controls reported activity that occurred at home and elsewhere, but they did not participate in organized exercise. During the subsequent 3-month observation, those who had completed the rehabilitation protocol did no more physical activity than those in the control group. Despite recommendations to maintain exercise at home, it is clearly apparent from our data that rehabilitation subjects had not done this, indicating that participation in a formal program increased compliance.

This pulmonary rehabilitation program aimed primarily at increasing endurance and strength improved daily physical activity and quality of life of patients with IPF. We assessed both using standardized and previously validated questionnaires, which captured the subjects' daily levels of physical activity (IPAQ) and their perceived health and symptoms (SGRQ-I).7,25 Physical activity is limited in IPF patients by dyspnea and fatigue, which may be worsened by muscular weakness. Physical activity in IPF is also limited by severe oxygen desaturation, which requires high levels of supplemental oxygen. Although the ultimate level of physical activity may in part be culturally or behaviorally determined, it is an important determinant of survival in patients with COPD. It is thus reasonable and consistent with our results that patients with IPF would likewise benefit from increased levels of daily physical activity. ${ }^{26}$

Subjective quality of life is dependent on many factors, including functional status and symptoms. Both the SF-36 and the SGRQ are useful in assessing quality of life in patients with IPF. Dyspnea significantly influences a number of the SF-36 components. ${ }^{28}$ Other factors, including mobility, social relationships, leisure activities, and energy levels, are reflected in questionnaire scores and represent important components of the quality of life. ${ }^{28}$

Changes in the SGRQ domain scores reflect important changes in the clinical status of individuals with IPF, and minimum important differences are represented by changes of 5-8 points in the SGRQ score. ${ }^{7,26} \mathrm{We}$ used a version of the SGRQ that is specific for patients with IPF (SGRQ-I), which retains the most reliable items for assessing health status. ${ }^{7}$ Pulmonary rehabilitation significantly improved symptoms as assessed by the SGRQ-I and clearly augmented self-reported physical activity as reflected by the IPAQ, indicating that pulmonary rehabilitation can improve quality of life. ${ }^{29}$

This investigation is limited by a number of issues related to the clinical problem and the use of subjective instruments to accrue data. First, because IPF is a rare disease $(<200,000$ cases in the United States $)$ and because of our use of a strict case definition, the final sample size was small. Sufficient power was demonstrably 
achieved in this pilot study to assess changes in the SGRQ-I for the symptoms domain and physical activity levels as assessed in the IPAQ. Questionnaires are innately subjective and prone to errors in recall and responses. The IPAQ does not correlate well with objective measures of physical activity. ${ }^{6}$ Our design applied the IPAQ identically to the control and rehabilitation groups, and we do not believe the validity of responses would differ systematically between groups. We also used the IPAQ as a repeated measure, as recommended. ${ }^{6}$ Subjects in this study were often limited during exercise training by severe hypoxemia (not fully corrected by high-flow supplemental oxygen) and dyspnea, so that the intensity of training may have been insufficient to produce maximum improvement.

We calculated the area under the cumulative MET-min (as reported weekly for 3 months) curve to generate the total MET-min exerted over the 3-month interval. This depends on self-reported recollection of physical activity levels, and is subject to recall variability and highly subjective assessments of the physical activity levels. Nonetheless, these data confirm a significant increase in physical activity during rehabilitation, and also show that this does not persist after pulmonary rehabilitation has been completed. A rehabilitation program that continues indefinitely might be of more value in maintaining increased physical activity.

\section{Conclusions}

In summary, we found that pulmonary rehabilitation benefits patients with IPF by enhancing their level of physical activity while in the program and by significantly reducing the disease's symptom burden. Such results should spur further multicenter investigations toward specific exercise therapy for patients with IPF and other interstitial lung diseases.

\section{ACKNOWLEDGMENTS}

We thank the subjects who participated in this study, and we appreciate the support of their families. We thank Nicole Eustis PT for participation in the rehabilitation program at its outset.

\section{REFERENCES}

1. Bourjeily G, Rochester C. Exercise training in chronic obstructive pulmonary disease. Clin Chest Med 2000;21(4):763-781.

2. Spruit MA, Singh SJ, Garvey C, ZuWallack R, Nici L, Rochester C, et al. An official ATS/ERS statement: key concepts and advances in pulmonary rehabilitation. Am J Respir Crit Care Med 2013;188(8): e13-e64.

3. King TE Jr, Pardo A, Selman M. Idiopathic pulmonary fibrosis. Lancet 2011;378(9807):1949-1961.

4. Holland A, Hill C. Physical training for interstitial lung disease. Cochran Database Syst Rev 2008(4):CD006322.
5. Nishiyama O, Taniguchi H, Kondoh Y, Kimura T, Ogawa T, Watanabe F, Nishimura K. Health related quality of life in patients with IPF: what is the main contributing factor? Resp Med 2005;99(4): 408-414.

6. Lee P, Macfarlane D, Tam T, Stewart S. Validity of the international physical activity questionnaire short form (IPAQ-SF): a systematic review. Int J Behav Nutr Physical Activity 2011;8(1):115-125.

7. Yorke J, Jones PW, Swigris JJ. Development and validity testing of an IPF specific version of the St George's Respiratory Questionnaire. Thorax 2010;65(10):921-926.

8. Raghu G, Collard HR, Egan JJ, Martinez FJ, Behr J, Brown KK, et al. An official ATS/ERS/JRS/ALAT statement; idiopathic pulmonary fibrosis: evidence-based guidelines for diagnosis and management. Am J Respir Crit Care Med 2011;183(6):788-824.

9. Jackson RM, Glassberg MK, Ramos CF, Bejarano PA, Butrous G, Gómez-Marin O. Sildenafil therapy and exercise tolerance in idiopathic pulmonary fibrosis: a randomized, controlled trial. Lung 2010; 188(2):115-123.

10. Jackson R, Ramos C, Gupta C, Gómez-Marin O. Exercise decreases plasma antioxidant capacity and increases urinary isoprostanes in IPF. Respir Med 2010;104(12):1919-1928.

11. Arcasoy SM, Christie JD, Ferrari VA, Sutton MS, Zisman DA, Blumenthal NP, et al. Echocardiographic assessment of pulmonary hypertension in patients with advanced lung disease. Am J Respir Crit Care Med 2003;167(5):735-740.

12. Lynch DA, Godwin JD, Safrin S, Starko KM, Hormel P, Brown KK, et al. High-resolution computed tomography in idiopathic pulmonary fibrosis: diagnosis and prognosis. Am J Respir Crit Care Med 2005; 172(4):488-493.

13. ATS Committee on Proficiency Standards for Clinical Pulmonary Function Laboratories. ATS statement: guidelines for the six-minute walk test. Am J Respir Crit Care Med 2002;166(1):111-117.

14. Dyspnea. Mechanisms, assessments and management: a consensus statement. Am J Respir Crit Care Med 1999;159(1):321-340.

15. Byrne NM, Hills AP, Hunter GR, Weinsier RL, Schutz Y. Metabolic equivalent: one size does not fit all. J Appl Physiol 2005;99(3):11121119.

16. Jones N, Killian K. Exercise limitation in health and disease. N Engl J Med 343(9):2000:632-641.

17. Cnaan A, Laird N, Slasor P. Mixed models: using the general linear mixed model to analyze unbalanced repeated measures and longitudinal data. Statistics Med 1997;16(20):2349-2380.

18. Bolton C, Bevan-Smith E, Blakely J, Crowe P, Elkin S, Garrod R, et al. British Thoracic Society guideline on pulmonary rehabilitation in adults. Thorax 2013;689(Suppl 2):ii1-ii30.

19. De Vries J, Seebregts A, Drent M. Assessing health status and quality of life in idiopathic pulmonary fibrosis: which measure should be used? Resp Med 2000;94(3):273-278.

20. Tomiko H, Imanaka K, Hashimoto K, Iwasaki H. Health-related quality of life in patients with IPF: cross-sectional and longitudinal study. Int Med 2007;46(18):1533-1542.

21. Han M, Swigris J, Liu L, Bartholmai B, Murray S, Giardino N, et al. Gender influences health-related quality of life in IPF. Resp Med 2010(5):104:724-730.

22. Ferreira A, Garvey C, Connors G, Hilling L, Rigler J, Ferrell S, et al. Pulmonary rehabilitation in interstitial lung disease: benefits and predictors of response. Chest 2009(2):135:442-447.

23. Nishiyama O, Kondoh Y, Kimura T, Kato K, Kataoke K, Ogawa T, et al. Effects of pulmonary rehabilitation in patients with idiopathic pulmonary fibrosis. Respirology 2008(3):13:354-399.

24. Salhi B, Troosters T, Behaegel M, Joos G, Derom E. Effects of pulmonary rehabilitation in patients with restrictive lung diseases. Chest 2010(2):137:273-279. 


\section{REHABILITATION AND QUALITY OF LIFE IN IPF}

25. Swigris J, Brown K, Behr J, du Bois R, King T, Raghu G, Wambolt F. The SF-36 and SGRQ: validity and first look at minimum important differences in IPF. Resp Med 2010;104(2):296-304.

26. Bajwah S, Ross JR, Peacock JL, Higginson IJ, Wells AU, Patel AS, et al. Interventions to improve symptoms and quality of life of patients with fibrotic interstitial lung disease: a systematic review of the literature. Thorax 2013;68(9):867-879.

27. Martinez T, Pereira C, dos Santos M, Ciconelli R, Guimaraes S,
Martinez J. Evaluation of the short-form 36-item questionnaire to measure health related quality of life in patients with IPF. Chest 2000(6):117:1627-1632.

28. De Vries J, Kessels B, Drent M. Quality of life in IPF patients. Eur Resp J 2001;17(5):954-961.

29. Swigris JJ, Kuschner WG, Jacobs SS, Wilson SR, Gould MK. Healthrelated quality of life in patients with IPF: a systematic review. Thorax 2005;60(7):588-594. 